\title{
Breaking and Flowering: The Budding Story of Macadamia ${ }^{\dagger}$
}

\author{
Francois Barbier 1,*, Ye Gong 1, Carola Parfitt 2, Craig Hardner ${ }^{3}$, John Wilkie ${ }^{4}$ and \\ Christine Beveridge 1
}

1 School of Biological Sciences, The University of Queensland, Saint Lucia 4072, Australia; ye.gong@uq.net.au (Y.G.); c.beveridge@uq.edu.au (C.B.)

2 Department of Agriculture and Fisheries, Bundaberg 4670, Australia; Siegrid.Parfitt@daf.qld.gov.au

3 Queensland Alliance for Agriculture and Food Innovation, The University of Queensland, Saint Lucia 4072, Australia; c.hardner@uq.edu.au

4 Wollongbar Primary Industries Institute, Department of Agriculture and Fisheries, Wollongbar 2477, Australia; John.Wilkie@daf.qld.gov.au

* Correspondence: f.barbier@uq.edu.au

† Presented at the third International Tropical Agriculture Conference (TROPAG 2019), Brisbane, Australia, 11-13 November 2019.

Published: 7 April 2020

\begin{abstract}
Macadamia is the only Australian native crop tree. Unlike some model crop trees like apple or peach trees, the understanding of the physiological mechanisms regulating bud break and flowering in macadamia has never been investigated. We have recently attempted to fill this gap by improving the genetic resources available for macadamia. We identified several components involved in flowering and bud break, including the FLOWERING LOCUS T (FT). FT is a mobile protein, synthesized in the leaves which triggers flowering in terminal and axillary buds. In macadamia, we found two FT-related genes, FTa and FTb namely. FTa and FTb are differentially regulated; $F T a$ being more abundant in axillary buds than leaves, which is unexpected based on the studies performed in model plants. Analysis of the promoter sequences of $F T a$ and $F T b$, revealed that the $F T a$ regulation may be closer to $F T$ regulation in the model plant Arabidopsis thaliana. Gene expression in buds revealed that the expression of TERMINAL FLOWER1 (TFL1), a gene previously reported to inhibit flowering, was induced prior to bud break and flowering. The patterns of marker gene expression in buds highlighted that the bud dormancy decreased before May, after which flowering induction occurs. Altogether these results shed light on the regulation of bud break and flowering in macadamia and demonstrate that some regulatory mechanisms may be different from other models.
\end{abstract}

Keywords: macadamia; flowering regulation; bud break; FLOWERING LOCUS T

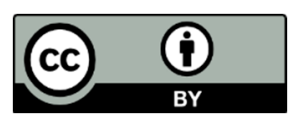

(C) 2020 by the authors. Licensee MDPI, Basel, Switzerland. This article is an open access article distributed under the terms and conditions of the Creative Commons Attribution (CC BY) license (http://creativecommons.org/licenses/by/4.0/). 